\title{
Identifier-Based Adaptive Robust Control for Servomechanisms With Improved Transient Performance
}

\author{
Guozhu Zhang, Jie Chen, Member, IEEE, and Zhiping Li
}

\begin{abstract}
This paper focuses on the adaptive robust control (ARC) for servomechanisms whose dynamic models are subject to unknown parameters, disturbance, and parameter sudden changes. To improve the control performance of the traditional ARC, a novel identifier-based ARC (IFARC) scheme is proposed. In this scheme, an identifier is utilized to accelerate the parameter tuning process and to heighten the accuracy of parameter estimation. A switching logic component based on a given performance index is introduced to select the better parameter estimate vector from those provided by the identifier and the adaptation law. As a result, transient performance can be improved according to the certainty equivalence principle. In addition, the exact reconstruction of the unknown parameters and exponential decay of the tracking error can be achieved under certain conditions. The stability and performance of IFARC are theoretically analyzed. Finally, simulation results show that the IFARC can achieve favorable tracking performance.
\end{abstract}

Index Terms-Adaptive control, identification, robust control, servomechanism.

\section{INTRODUCTION}

$\mathbf{W}$ HEN the parameters of a servomechanism are not exactly known or are totally unknown, adaptive robust control (ARC) can be utilized to achieve high performance (i.e., fast transient performance and asymptotic tracking) [1]-[3]. However, since the traditional ARC can only "learn" gradually from the plant states, it may cost a long period of time to achieve the desired output tracking performance [4]. Furthermore, the traditional adaptive robust controllers are based on the assumption that the plant parameters are constant, which is not guaranteed in many cases. For example, when a robot manipulator picks up an object, its payload jumps [5], [6]. If the load changes suddenly in a servomechanism, the traditional ARC may lead to a long-time transient response and a large output tracking error. That is because the persistence of excitation (PE) condition [7], which assures the convergence rate of parameter estimates, is not satisfied in many applications. In addition, the traditional ARC can only guarantee that the tracking error

Manuscript received February 27, 2009; revised October 13, 2009; accepted October 13, 2009. Date of publication November 6, 2009; date of current version June 11, 2010. This work was supported by the National Science Fund for Distinguished Young Scholar under Grant 60925011.

The authors are with the Beijing Key Laboratory of Automatic Control System, School of Automation, Beijing Institute of Technology, Beijing 100081, China (e-mail: chenjie@bit.edu.cn).

Color versions of one or more of the figures in this paper are available online at http://ieeexplore.iee.org.

Digital Object Identifier 10.1109/TIE.2009.2035461 converges exponentially to a small neighborhood of the origin and converges asymptotically to zero with unknown settling time [8].

According to the certainty equivalence principle [9], if the parameter tuning time could be shortened and the unknown parameters could be reconstructed, the output tracking error would be improved. To accelerate the adaptation process and improve the transient performance of adaptive control, a multiple-model adaptive control (MMAC) is proposed [10]. It includes several fixed models and adaptive models, which would be selected to represent the dynamics of the plant [11], [12]. Its transient performance and parameter estimation can be greatly improved by the switching among multiple models. If more models were embedded in MMAC, the improvement would be more notable. However, a large number of models will make the controller rather complicated and cause difficulties in engineering application.

The control problem of servomechanisms subjected to unknown parameters, disturbances, and parameter sudden changes will be considered in this paper. For such kind of plants, their transient performance would be badly deteriorated by the sudden parameter changes, which has never been considered in the ordinary adaptive robust controller design. Thus, this is a challenging control problem. Our motivation to study this problem is to improve the performance of the servomechanism with sudden parameter changes and to extend the application range of $A R C$.

To solve this problem, we propose in this paper a novel identifier-based ARC (IFARC) scheme, which adopts an identifier instead of multiple models to accelerate the adaptation process. This method is less complicated than the multiplemodel schemes. However, in fact, the identifier is equivalent to an infinite number of models; thus, the IFARC has better transient performance and parameter estimation than the MMAC. With IFARC, the unknown parameters of the servomechanism can be reconstructed in finite time under certain conditions, and then, the desirable transient performance of the system can be achieved. In addition, the IFARC can also detect the parameter jumps online effectively. Due to the aforementioned characteristics, the IFARC is applicable to the servomechanisms subjected to load jumps.

The main contributions of this paper are as follows: 1) We proposed a novel architecture of the original identifier-based adaptive robust controller; 2) we put forward a new method to improve transient performance and to deal with parameter 
sudden changes for the adaptive control system; and 3) the stability proof and performance analysis of the proposed IFARC are given.

This paper is organized as follows. The problem description is presented in Section II. The design procedure of the proposed IFARC is provided in Section III. The stability proof and performance analysis are given in Section IV. Simulations are described and analyzed in Section V, and conclusions are drawn in Section VI.

\section{Dynamic Models And PRoblem Formulations}

\section{A. Dynamic Model of Servomechanisms}

Without loss of generality, the mechanical and electrical dynamics of a de servomechanism can be described as [13]

$$
\begin{aligned}
J \ddot{q}+T_{f}+T_{l}+T_{\text {dis }} & =T_{m} \\
K_{E} \dot{q}+L_{a} \frac{d I_{a}}{d t}+R_{a} I_{a} & =u \\
T_{m} & =K_{T} I_{a}
\end{aligned}
$$

where $q$ denotes the output angle; $J, T_{f}, T_{l}, T_{\text {dis }}$, and $T_{m}$ denote the mechanical parameters: inertia, friction torque, load torque, disturbance torque, and generated torque, respectively; $u, I_{a}, R_{a}$, and $L_{a}$ denote the electrical parameters: input voltage, armature current, armature resistance, and armature inductance, respectively; $K_{T}$ denotes the electrical-mechanical energy conversion constant; and $K_{E}$ denotes the back electromotive force coefficient. In engineering practice, the friction torque $T_{f}$ is often described by the Coulomb plus viscous model given as follows [14]:

$$
T_{f}(\dot{q})=T_{c} \operatorname{sgn}(\dot{q})+B \dot{q}
$$

where $T_{c}$ represents the level of Coulomb friction torque, $B$ represents the viscous friction coefficient, and sgn denotes the signum function.

From (1) and (4), we have

$$
J \ddot{q}+B \dot{q}=T_{m}-T_{c} \operatorname{sgn}(\dot{q})-T_{l}-T_{\text {dis }} .
$$

In general, the electrical constant $L_{a} / R_{a}$ is small (compared to the mechanical time constant $J / B$ ); therefore, from (2), (3), and (5), we know that the electrical transients decay very rapidly and $L_{a}\left(d I_{a} / d t\right)$ is very close to zero [15]. Thus, the dynamics of a servomechanism can be simplified as [13]

$$
\ddot{q}=\frac{K_{1}}{J} u-\frac{K_{2}}{J} \dot{q}-\frac{T_{c}}{J} \operatorname{sgn}(\dot{q})-\frac{1}{J}\left(T_{l}+T_{\text {dis }}\right)
$$

where

$$
K_{1}=K_{T} / R_{a} \quad K_{2}=\left(K_{T} K_{E}+B R_{a}\right) / R_{a} .
$$

Here, the output angle $q$ is regarded as the system output $y$. Defining the output angle and angular velocity as the state variables, i.e., $\left[x_{1}, x_{2}\right]^{\mathrm{T}}=[q, \dot{q}]^{\mathrm{T}}$, from (6), the entire system can be expressed in the state space form as

$$
\left\{\begin{array}{l}
\dot{x}_{1}=x_{2} \\
\dot{x}_{2}=\frac{1}{J}\left(K_{1} u-K_{2} x_{2}-T_{c} S_{f}-T_{l}-T_{\text {dis }}^{\prime}\right) \\
y=x_{1}
\end{array}\right.
$$

where $T_{\text {dis }}^{\prime}=T_{\text {dis }}+T_{c}\left(\operatorname{sgn}\left(x_{2}\right)-S_{f}\right)$, and $S_{f}$ is a continuous function used to approximate $\operatorname{sgn}\left(x_{2}\right)$. It is defined as

$$
S_{f}=2 / \pi \arctan \left(K_{s} x_{2}\right), \quad K_{s}>0
$$

where $K_{s}$ is a positive constant to be chosen. It should be selected sufficiently large, so that $S_{f}$ can be adequately close to $\operatorname{sgn}\left(x_{2}\right)$. Define $T_{n}$ as the mean value of $T_{l}+T_{\text {dis }}^{\prime}$ and $d$ as the offset value, i.e., $d=T_{n}-T_{l}-T_{\text {dis }}^{\prime}$. Then, from (8), we have

$$
\left\{\begin{array}{l}
\dot{x}_{1}=x_{2} \\
\dot{x}_{2}=\theta_{1} u-\theta_{2} x_{2}-\theta_{3} S_{f}-\theta_{4}+\Delta \\
y=x_{1}
\end{array}\right.
$$

where $\Delta=d / J\left(\mathrm{rad} / \mathrm{s}^{2}\right)$, and $\theta_{i}(i=1, \ldots, 4)$ is given as

$$
\begin{aligned}
\theta_{1} & =\frac{K_{1}}{J}\left(\left(\mathrm{rad} / \mathrm{s}^{2}\right) / \mathrm{V}\right) & \theta_{2} & =\frac{K_{2}}{J}(1 / \mathrm{s}) \\
\theta_{3} & =\frac{T_{c}}{J}\left(\mathrm{rad} / \mathrm{s}^{2}\right) & \theta_{4} & =\frac{T_{n}}{J}\left(\mathrm{rad} / \mathrm{s}^{2}\right) .
\end{aligned}
$$

We assume that the parameters are unknown and may change suddenly from one value to another.

Remark 1: From (10), we observe that the additive measurement noises on $x_{2}$ and $S_{f}$ can be lumped into the term $\Delta$. In fact, the additive measurement noise on $x_{1}$ in the closed-loop system could be also lumped into $\Delta$, as will be illustrated in Section III. Hence, $\Delta$ can be regarded as a lumped disturbance term, which does not only represent external disturbances but also additive sensor noises.

\section{B. Assumptions and Problem Formulations}

For simplicity, the following notations will be used: $\bullet{ }_{i}$ for the $i$ th component of the vector $\bullet, \hat{\bullet}$ for the estimate of $\bullet, \bullet$ min for the minimum value of $\bullet$, and $\bullet$ max for the maximum value of $\bullet$. The operation $\leqslant$ for two vectors is performed in terms of the corresponding elements of the vectors.

In general, the parameters of the model cannot be accurately determined. Thus, we assume that the uncertain parameters are in certain known intervals, as shown in Assumptions 1 and 2. In addition, Assumption 3 is made for the desired motion trajectory $x_{1 d}(t)$.

Assumption 1: $\theta_{i} \in\left[\theta_{i \min }, \theta_{i \max }\right], \quad i=1, \ldots, 4$, where $\theta_{i \text { min }}$ and $\theta_{i \max }$ are known. Moreover, $\theta_{1 \text { min }}>0$, which conforms to the physical point of view.

Assumption 2: The lumped disturbance $\Delta$ in (10) is bounded, i.e., $|\Delta| \leqslant \delta$, where $\delta$ is known.

Assumption 3: The desired trajectory $x_{1 d}$ is continuous with its first-order derivative $\dot{x}_{1 d}$ and second-order derivative $\ddot{x}_{1 d}$ bounded and available.

Consider model (10) which has unknown parameters, disturbance, and parameter jumps. The control problem of this paper can be stated as follows: Given the desired motion trajectory $x_{1 d}(t)$, the objective is to synthesize a control action $u$ such that the system output $y=x_{1}$ tracks $x_{1 d}(t)$ as closely as possible in spite of various model uncertainties. 


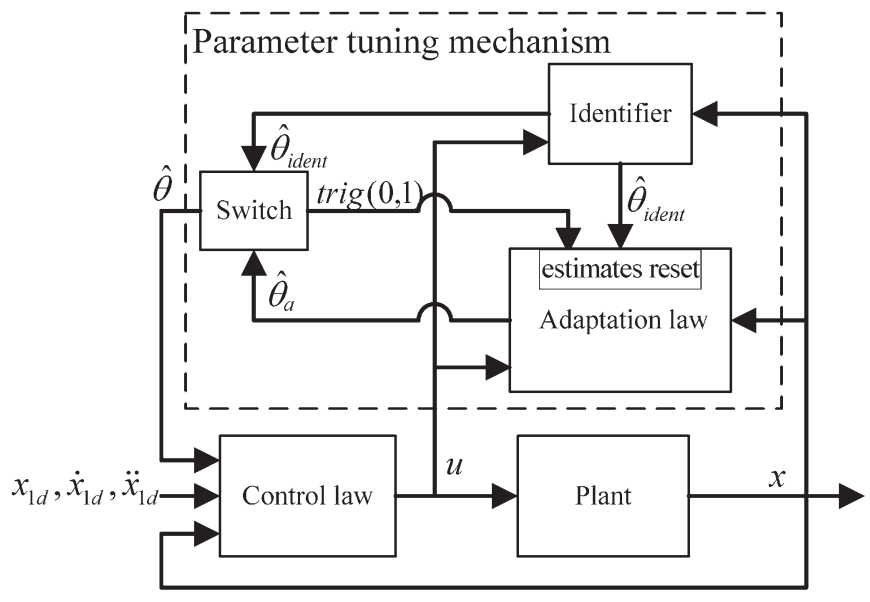

Fig. 1. Architecture of the IFARC.

\section{IFARC}

Through parameter adaptation, traditional ARC can reduce the adverseness of parametric uncertainties for the system. Nevertheless, the adaptation law can only tune parameters gradually, which may result in a long period to achieve the desired control performance. When the plant parameters jump from one value to another, sluggish transient response and large tracking error may appear.

According to the certainty equivalence principle [16], if parameters converge to their actual values in finite time, the tracking performance of the ARC system can be greatly improved. Motivated by this fact and by the recent work in [17], we propose a novel ARC scheme based on identifier in this paper. This method assures the exact reconstruction of the unknown parameters in finite time and the exponential convergence of the tracking error to zero, provided that the given condition is satisfied. It can be seen later that this condition is less rigorous than the PE condition, so that it is more accommodated to engineering applications. In this section, we first describe the architecture of the proposed ARC and then expound the synthesis procedures in details.

\section{A. Controller Architecture}

The identifier-based adaptive robust controller has a similar architecture as the multiple-model adaptive controller [11], [12]. However, the proposed controller does not possess multiple fixed models or adaptive models; instead, it has an identifier. The architecture of IFARC is shown in Fig. 1. As shown, the IFARC consists of four parts, i.e., control law, adaptation law, identifier, and switching logic. The last three components constitute the parameter tuning mechanism. These four parts have different functionalities as follows: 1) The control law produces control action to the plant, and it is dependent on the states $x_{1}$ and $x_{2}$, the input trajectories $x_{1 d}, \dot{x}_{1 d}$, and $\ddot{x}_{1 d}$, and the parameter estimate $\hat{\theta} ; 2$ ) the identifier yields a parameter estimate $\hat{\theta}_{\text {ident }}$ by online identification; 3 ) the adaptation law produces parameter estimate $\hat{\theta}_{a}$ through adaptation, and the estimate $\hat{\theta}_{a}$, which is also the state vector of the adaptation law, will be reset to $\hat{\theta}_{\text {ident }}$, if trig $=1$ and some other conditions are satisfied, where trig $=1$ implies that $\hat{\theta}_{\text {ident }}$ is better than $\hat{\theta}_{a}$ according to a certain performance index; and 4) the switching logic has the functionality of selecting the better estimate from $\hat{\theta}_{\text {ident }}$ and $\hat{\theta}_{a}$ to be $\hat{\theta}$, according to the performance index. Then, $\hat{\theta}$ is employed by the control law. In the following sections, the design procedures of IFARC will be described in details.

\section{B. Control Law}

The control law design follows the ARC synthesis procedure proposed in [8] and [18]. First, define a switching-function-like quantity [19] as

$$
z_{2}=\dot{e}_{1}+k_{p} e_{1}=x_{2}-x_{2 \mathrm{eq}}, \quad x_{2 \mathrm{eq}} \triangleq \dot{x}_{1 d}-k_{p} e_{1}
$$

where $e_{1}=x_{1}-x_{1 d}(t)$ is the output tracking error, and $k_{p}$ is any positive feedback gain. Denote the Laplace transform of $e_{1}(t)$ and $z_{2}(t)$ as $E_{1}(s)$ and $Z_{2}(s)$, respectively. Since $G_{s}(s)=E_{1}(s) / Z_{2}(s)=1 /\left(s+k_{p}\right)$ is a stable transfer function, if $z_{2}(t)$ is small or converges to zero exponentially, then the output tracking error $e_{1}(t)$ will be small or converges to zero exponentially. Differentiating (12) and noting (10), we have

$$
\dot{z}_{2}=\dot{x}_{2}-\dot{x}_{2 \mathrm{eq}}=\theta_{1} u-\theta_{2} x_{2}-\theta_{3} S_{f}-\theta_{4}+\Delta-\dot{x}_{2 \mathrm{eq}}
$$

where $\dot{x}_{2 \mathrm{eq}}=\ddot{x}_{1 d}-k_{p} \dot{e}$. For simplicity, we define vectors $\bar{x}$ and $\bar{x}_{1 d}$ as $\bar{x}=\left[x_{1}, x_{2}\right]^{\mathrm{T}}$ and $\bar{x}_{d}=\left[x_{1 d}, \dot{x}_{1 d}, \ddot{x}_{1 d}\right]^{\mathrm{T}}$, respectively. From (13), we can synthesize the control law according to the ARC approach. The control input $u$ consists of two parts, given by

$$
\left\{\begin{array}{l}
u=u_{a}\left(\bar{x}, \hat{\theta}, \bar{x}_{d}\right)+u_{s}\left(\bar{x}, \bar{x}_{d}\right) \\
u_{a}=\frac{1}{\hat{\theta}_{1}}\left(\hat{\theta}_{2} x_{2}+\hat{\theta}_{3} S_{f}+\hat{\theta}_{4}+\dot{x}_{2 \mathrm{eq}}\right) \\
u_{s}=u_{s 1}+u_{s 2}, \quad u_{s 1}=-\frac{k_{s 1}}{\theta_{1 \min }} z_{2}
\end{array}\right.
$$

where $k_{s 1}>0$ is a controller parameter to be chosen, $u_{a}$ is an adaptive control term, and $u_{s}$ is a robust control term consisting of two parts, i.e., $u_{s 1}$ and $u_{s 2}$. The robust control function $u_{s 2}$ should be selected to satisfy

$$
\begin{aligned}
z_{2} u_{s 2} & \leq 0 \\
z_{2}\left(\theta_{1} u_{s 2}-\varphi^{\mathrm{T}} \tilde{\theta}+\Delta\right) & \leq \varepsilon
\end{aligned}
$$

where $\varphi^{T}=\left[u_{a},-x_{2},-S_{f},-1\right], \tilde{\theta}=\hat{\theta}-\theta$, and $\varepsilon>0$ is a design parameter. There are many ways to design $u_{s 2}$ satisfying (15) and (16). One of them is to let

$$
u_{s 2}=-\frac{h_{2}}{2 \theta_{1 \min } \varepsilon} z_{2}
$$

where $h_{2}$ is any function or constant satisfying $h_{2} \geq \| \theta_{\max }-$ $\theta_{\min }\left\|^{2}\right\| \varphi \|^{2}+\delta^{2}$. Readers can refer to [8] and [20] for other choices of $u_{s 2}$. 
From (12)-(14), we know that the closed-loop dynamics can be represented by the following:

$$
\begin{aligned}
\dot{z}_{2}= & -\varphi^{\mathrm{T}} \tilde{\theta}+\theta_{1} u_{s 2}-k_{s 1} \frac{\theta_{1}}{\theta_{1 \min }} z_{2}+\Delta \\
= & -k_{s 1} \frac{\theta_{1}}{\theta_{1 \min }}\left(x_{2}+k_{p} x_{1}-\dot{x}_{1 d}-k_{p} x_{1 d}\right) \\
& -\varphi^{\mathrm{T}} \tilde{\theta}+\theta_{1} u_{s 2}+\Delta .
\end{aligned}
$$

From this equation, we can see that the additive measurement noises on $x_{1}$ could be lumped into $\Delta$. Hence, the additive noise on the system's output can be taken into account, as indicated in Remark 1.

\section{Parameter Tuning Mechanism}

As shown in Fig. 1, the parameter tuning mechanism of IFARC consists of three components, i.e., adaptation law, identifier, and switching logic. The motivation of this mechanism is to utilize the identifier to improve the parameter estimation of adaptation law. In this section, we first describe the development of the three components in details and then expound the parameter tuning scheme.

1) Adaptation Law: The discontinuous projection operator used in the adaptation law is defined as $\operatorname{Proj}_{\hat{\theta}_{a}}(\bullet)=$ $\left[\operatorname{Proj}_{\hat{\theta}_{a 1}}(\bullet), \ldots, \operatorname{Proj}_{\hat{\theta}_{a 4}}\left(\bullet_{4}\right)\right]^{\mathrm{T}}$, where

$$
\operatorname{Proj}_{\hat{\theta}_{a i}}\left(\bullet_{i}\right)= \begin{cases}0, & \text { if } \hat{\theta}_{a i}=\theta_{i \max } \text { and } \bullet_{i}>0 \\ 0, & \text { if } \hat{\theta}_{a i}=\theta_{i \text { min }} \text { and } \bullet_{i}<0 \\ \bullet_{i}, & \text { otherwise. }\end{cases}
$$

The adaptation law is given by

$$
\begin{cases}\hat{\theta}_{a}(t)=\hat{\theta}_{\text {ident }}(t), & \text { if } t=t_{i}(i=0,1, \ldots) \text { and } \text { trig }=1 \\ \dot{\hat{\theta}}_{a}(t)=\operatorname{Proj}_{\hat{\theta}_{a}}(\Gamma \tau), & \text { else }\end{cases}
$$

where $t_{0}=0, t_{i}(i=1,2, \ldots)$ denotes the estimation reset instants depending on the integral of a regressor matrix to be synthesized later, trig is the trigger signal given by the switching logic (the trigger signal is effective when $\hat{\theta}_{\text {ident }}$ is better than $\hat{\theta}_{a}$ according to a certain performance index), $\Gamma$ is a diagonal positive definite matrix, and $\tau$ is an adaptation function to be given.

It should be noted that, in (18), the parameter estimates of adaptation law are reset to the better estimates given by the identifier, only when the trigger signal is in effect. Loosely speaking, the parameter estimation in adaptation law will be improved by embedding an identifier into the controller. More details about this improvement will be shown later.

For any adaptation function $\tau$, the projection mapping used in (18) assures the following properties [21].

Property 1:

$$
\left(\hat{\theta}_{a}-\theta\right)^{\mathrm{T}}\left(\Gamma^{-1} \operatorname{Proj}_{\hat{\theta}_{a}}(\Gamma \tau)-\tau\right) \leq 0 \quad \forall \tau .
$$

Property 2: If $\hat{\theta}_{a}\left(t^{\prime}\right) \in \Omega_{\theta} \triangleq\left\{\hat{\theta}: \theta_{\min } \leq \hat{\theta} \leq \theta_{\max }\right\}$ and the adaptation law is $\dot{\hat{\theta}}_{a}(t)=\operatorname{Proj}_{\hat{\theta}_{a}}(\Gamma \tau)$ in the time interval $\left[t^{\prime}, t^{\prime \prime}\right]$, then

$$
\hat{\theta}_{a}(t) \in \Omega_{\theta} \quad \forall t^{\prime} \leq t \leq t^{\prime \prime}
$$

where $\theta_{\min }=\left[\theta_{1 \min }, \theta_{2 \min }, \theta_{3 \min }, \theta_{4 \min }\right]^{\mathrm{T}}, \quad$ and $\theta_{\max }=$ $\left[\theta_{1 \text { max }}, \theta_{2 \text { max }}, \theta_{3 \text { max }}, \theta_{4 \text { max }}\right]^{\mathrm{T}}$.

In order to develop the adaptation law and identifier, the regression form of system (10) should be first constructed. Define a regressor $\varphi_{20}=\left[u,-x_{2},-S_{f},-1\right]^{\mathrm{T}}$. Let $\varphi_{20}$ pass through low-pass filters with state resets, and then, we have

$$
\begin{gathered}
\begin{cases}f_{u}(t)=0, & t=t_{i} \\
\kappa \dot{f}_{u}(t)+f_{u}(t)=u(t), & t \neq t_{i}\end{cases} \\
\begin{cases}f_{x_{2}}(t)=0, & t=t_{i} \\
\kappa \dot{f}_{x_{2}}(t)+f_{x_{2}}(t)=x_{2}(t), & t \neq t_{i}\end{cases} \\
\begin{cases}f_{S_{f}}(t)=0, & t=t_{i} \\
\kappa \dot{f}_{S_{f}}(t)+f_{S_{f}}(t)=S_{f}(t), & t \neq t_{i}\end{cases} \\
\begin{cases}f_{1}(t)=0, & t=t_{i} \\
\kappa \dot{f}_{1}(t)+f_{1}(t)=1, & t \neq t_{i}\end{cases}
\end{gathered}
$$$$
t=t_{i}(i=0,1, \ldots)
$$

where $\kappa$ is generally a small positive number, such that the filter outputs can track their corresponding inputs closely. From (10) and (21a)-(21d), we know that, during the period between two adjacent state resets, the following regression form holds:

$$
\varphi_{2}^{\mathrm{T}} \theta+f_{\Delta}=\frac{x_{2}-f_{x_{2}}}{\kappa} \triangleq f_{\dot{x}_{2}}
$$

where $\varphi_{2}=\left[f_{u},-f_{x_{2}},-f_{S_{f}},-f_{1}\right]^{\mathrm{T}}$, and $f_{\Delta}$ is the filter output of $\Delta$ given by

$$
\begin{cases}f_{\Delta}(t)=0, & t=t_{i} ; \quad i=0,1, \ldots \\ \kappa \dot{f}_{\Delta}(t)+f_{\Delta}(t)=\Delta, & t \neq t_{i} .\end{cases}
$$

The quantity $f_{\Delta}$ can be viewed as the modeling error. Due to the boundedness of $\Delta, f_{\Delta}$ is also bounded. Furthermore, since the mean value of $\Delta$ is zero, the mean value of $f_{\Delta}$ is zero, too.

According to (22), we construct the estimate of $f_{\dot{x}_{2}}$ as

$$
\hat{f}_{\dot{x}_{2}}=\varphi_{2}^{\mathrm{T}} \hat{\theta}_{a} .
$$

Then, the estimation error $\varepsilon_{e}$ can be derived as

$$
\varepsilon_{e}=f_{\dot{x}_{2}}-\hat{f}_{\dot{x}_{2}}=-\varphi_{2}^{\mathrm{T}} \tilde{\theta}_{a}+f_{\Delta}
$$

where $\tilde{\theta}_{a}=\hat{\theta}_{a}-\theta_{a}$. Now, the gradient type adaptation law can be constructed. To realize this, the quantity $\tau$ in (18) is chosen as

$$
\tau=\frac{\varphi_{2}}{1+v \varphi_{2}^{\mathrm{T}} \varphi_{2}} \varepsilon_{e}
$$

where $v$ is a positive design parameter.

2) Identifier: Assuming that $f_{\Delta}=0$ and that $\theta$ does not change during $\left[t_{i}, t_{i+1}\right], \exists i \in\{0,1, \ldots\}$, from (22), we have

$$
\int_{t_{i}}^{t_{i+1}} \varphi_{2} \varphi_{2}^{\mathrm{T}} d r \theta=\int_{t_{i}}^{t_{i+1}} \varphi_{2} f_{\dot{x}_{2}} d r
$$


In order to accommodate sudden load changes, the identifier is constructed as follows:

$$
\begin{aligned}
& P=\left\{\begin{array}{l}
P_{0}, \\
\int_{t_{i}}^{t} \varphi_{2} \varphi_{2}^{\mathrm{T}} d r
\end{array}\right. \\
& t=t_{0} \\
& t_{i}<t \leq t_{i+1} ; \quad i=0,1, \ldots \\
& Q=\left\{\begin{array}{l}
Q_{0}, \\
t \\
\int_{t_{i}}^{t} \varphi_{2} f_{\dot{x}_{2}} d r
\end{array}\right. \\
& t=t_{0} \\
& t_{i}<t \leq t_{i+1} ; \quad i=0,1, \ldots \\
& \hat{\theta}_{\text {ident }}=P\left(t_{i}\right)^{-1} Q\left(t_{i}\right), \quad t_{i} \leq t<t_{i+1} ; \quad i=0,1, \ldots
\end{aligned}
$$

where $P_{0}$ and $Q_{0}$ satisfy $P_{0}^{-1} Q_{0} \in \Omega_{\theta}$. From (27)-(29), we know that the identifier resets its integrals to zero after the instants when $\hat{\theta}_{\text {ident }}$ is computed. Noting the right-hand side of (29), we can see that $\hat{\theta}_{\text {ident }}$ keeps constant during each interval $\left[t_{i}, t_{i+1}\right)$. The time instants $t_{i}(i=1,2, \ldots)$ are determined in the following way.

Let $t_{0}=0$; then, $t_{1}$ is the first instant when $P(t)$ satisfies the following.

Condition 1) $P(t)$ becomes invertible.

Condition 2) $P(t)^{-1} Q(t) \in \Omega_{\theta}$.

At instant $t_{1}, \hat{\theta}_{\text {ident }}$ is computed through (29). Moreover, from (27) and (28), $P(t)$ and $Q(t)$ are reset to zero matrix (i.e., all matrix entries are zero), so that the two matrices become not invertible. Similarly, $t_{2}$ is the first instant when $P(t)$ satisfies conditions 1 ) and 2) after $t_{1}$. In addition, the same treatment is done during $\left[t_{2}, t_{3}\right)$ as in $\left[t_{1}, t_{2}\right)$. Other instants (i.e., $t_{3}, t_{4}, \ldots$ ) are defined in the same way. It should be noted that the aforementioned conditions can be matched even when the PE condition [7] is not satisfied.

In order to check the invertibility of $P(t)$, the determinant of $P(t)$ is computed. As we know, the determinant of a matrix is equal to the product of all eigenvalues. Moreover, from (27), $P(t)$ is a positive semidefinite matrix. Thus, it is invertible if and only if all of its eigenvalues are positive, which is equal to the fact that the determinant of $P(t)$ is positive. To avoid numerical problem in inverse matrix computation, a small positive threshold value $\varepsilon_{t}$ is introduced to the invertibility judgment. Then, the condition on which the matrix $P(t)$ becomes invertible is

$$
\operatorname{det}(P(t)) \geq \varepsilon_{t}
$$

where $\operatorname{det}(P(t))$ denotes the determinant of $P(t)$. For the reason that $\operatorname{det}(P(t))$ is a continuous function of the entries of $P(t)$, which are continuous in $t$ for $t \in\left(t_{i}, t_{i+1}\right]$, it follows that $\operatorname{det}(P(t))$ is continuous in $t$ during $\left(t_{i}, t_{i+1}\right]$. Since $P(t)$ is reset to zero when conditions 1$)$ and 2 ) are satisfied, the time instant $t_{i}(i=1,2, \ldots)$ is the first instant satisfying (31) after $t_{i-1}$

$$
\left\{\begin{array}{l}
\operatorname{det}(P(t)) \geq \varepsilon_{t} \\
P(t)^{-1} Q(t) \in \Omega_{\theta} .
\end{array}\right.
$$

The reset will make $\operatorname{det}(P(t))$ become zero, and then, (31) will become unsatisfied; hence, the reset instants of $P(t)$ and $Q(t)$ (i.e., $t_{0}, t_{1}, t_{2}, \ldots$ ) are sparsely distributed on time axis. Through the resets in $P(t)$ and $Q(t)$, the identifier can estimate parameters only according to the recent data. Therefore, when parameters change suddenly, the past data before jump will not influence the estimation after the first reset subsequent to the jump.

3) Switching Logic: Noting that mean value of $f_{\Delta}$ is zero, from (23), we know that unbiased estimates would be obtained during $\left[t_{i+1}, t_{j}\right)(j>i+1)$ by the identifier, when no parameter jump occurs in $\left[t_{i}, t_{j}\right)$. However, in some time, unbiased estimates are not available. Specifically, if the parameters jump at the time instant $t_{J}$, then in the period between $t_{J}$ and the first reset after $t_{J}$, the identifier estimation will be affected by the data before $t_{J}$. In this case, the estimates of the identifier may be worse than those of the adaptation law. Thus, the switching logic is introduced to select the better parameter vector from $\hat{\theta}_{\text {ident }}$ and $\hat{\theta}_{a}$ as the controller parameter vector $\hat{\theta}$. In other words, if $\hat{\theta}_{\text {ident }}$ is better than $\hat{\theta}_{a}$ in the instant $t_{i}$, then $\hat{\theta}_{a}$ is reset to $\hat{\theta}_{\text {ident }}$; otherwise, the adaptation does not reset.

The following performance index is utilized by the switching logic component:

$$
J(\bullet, t)=\left(\bullet^{\mathrm{T}} \varphi_{2}(t)-f_{\dot{x}_{2}}(t)\right)^{2}+\gamma \int_{t-t_{e}}^{t}\left(\bullet^{\mathrm{T}} \varphi_{2}(p)-f_{\dot{x}_{2}}(p)\right)^{2} d p
$$

where $t_{e}$ is the length of the time interval for the performance index evaluation; $\gamma$ is a positive constant to be selected. The parameter estimate vector with smaller value of $J$ is considered as better. Subsequently, $\hat{\theta}$ satisfies

$$
\hat{\theta}(t)= \begin{cases}\hat{\theta}_{\text {ident }}(t), & J\left(\hat{\theta}_{\text {ident }}, t\right) \leq J\left(\hat{\theta}_{a}, t\right) \\ \hat{\theta}_{a}(t), & J\left(\hat{\theta}_{\text {ident }}, t\right)>J\left(\hat{\theta}_{a}, t\right) .\end{cases}
$$

The trigger signal trig is produced by

$$
\text { trig }= \begin{cases}1, & J\left(\hat{\theta}_{\text {ident }}, t\right) \leq J\left(\hat{\theta}_{a}, t\right) \\ 0, & J\left(\hat{\theta}_{\text {ident }}, t\right)>J\left(\hat{\theta}_{a}, t\right) .\end{cases}
$$

4) Parameter Tuning Scheme: In order to obtain estimates better than those acquired merely from the identifier or adaptation law, the three components (i.e., adaptation law, identifier, and switching logic) should work under the following parameter tuning scheme.

1) Initialization: Preselect parameter vectors $\hat{\theta}_{a}(0)$ and $\hat{\theta}_{\text {ident }}(0)$, which are both within the set $\Omega_{\theta}$, to be the initial value of the adaptation law and identifier, respectively.

2) Identifier reset: The matrices $P(t)$ and $Q(t)$ reset to zero, and the states of filter (21a)-(21d) reset to zero, when (31) is satisfied.

3) Adaptation reset: $\hat{\theta}_{a}$ is reset to $\hat{\theta}_{\text {ident }}$, when trig $=1$ and (31) is satisfied.

4) Switching logic: It is given by (33) and (34).

For clarity, we show a flow diagram of the entire parameter tuning scheme in Fig. 2. In this diagram, the variable $r$ is 


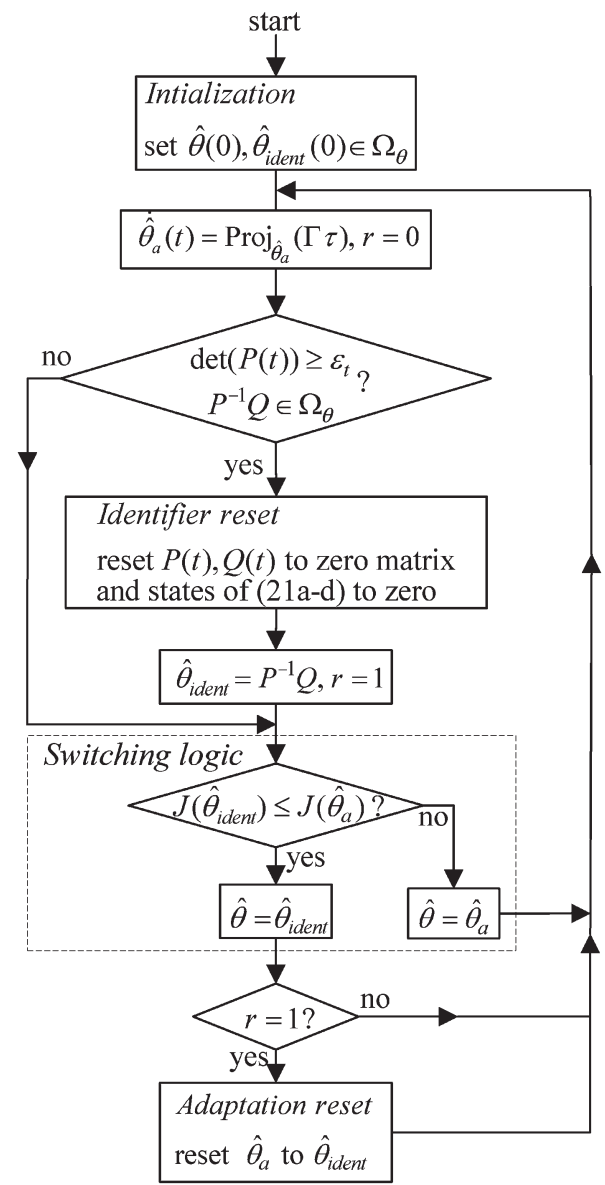

Fig. 2. Flow diagram of the parameter tuning scheme.

introduced to label the time instant $t_{i}$. Coherent with (18), if $r=1$ and $\operatorname{trig}=1$, then $\hat{\theta}_{a}$ will be reset to $\hat{\theta}_{\text {ident }}$.

\section{Stability Proof and Performance Analysis}

Theorem 1: Suppose that the identifier-based adaptive robust controller proposed in Section III is applied to the plant (10). Then, the controller guarantees the following.

1) The parameter vector $\hat{\theta}$ satisfies

$$
\hat{\theta}(t) \in \Omega_{\theta} \quad \forall t \geq 0 .
$$

2) The closed-loop system is globally stable. The positive definite function $V_{s}$ defined by

$$
V_{s}=\frac{1}{2} z_{2}^{2}
$$

is bounded above by

$$
V_{s} \leq \exp (-\lambda t) V_{s}(0)+\frac{\varepsilon}{\lambda}[1-\exp (-\lambda t)]
$$

where $\lambda=2 k_{s 1}$.

Proof: We first prove 1). From (29) and conditions 1) and 2), we have

$$
\hat{\theta}_{\text {ident }}(t) \in \Omega_{\theta} \quad \forall t \geq 0 .
$$

In the following, we are going to prove $\hat{\theta}_{a}(t) \in \Omega_{\theta}$ in two cases as in (18).

Case 1) $t=t_{i}(i=0,1, \ldots)$, and trig $=1$.

Noting (18), in this case

$$
\hat{\theta}_{a}=\hat{\theta}_{\text {ident }} .
$$

From (38), one has $\hat{\theta}_{a} \in \Omega_{\theta}$ in this case.

Case 2) $t \neq t_{i}(i=0,1,2, \ldots)$ or $t$ rig $\neq 1$.

From (18), we know that $\hat{\theta}_{a}$ is updated by $\dot{\hat{\theta}}_{a}(t)=$ $\operatorname{Proj}_{\hat{\theta}_{a}}(\Gamma \tau)$. Due to the sparse distribution of $t_{i}$, as described in (31), for any $t^{\prime \prime}$ belonging to case $2)$, there exists an instant $t_{k}(k \in N)$ such that no $t_{i}(i=0,1, \ldots)$ exists in $\left(t_{k}, t^{\prime \prime}\right]$. Let $t^{\prime}=t_{k}$. Noting the result in case 1), from Property 2 in Section III, we have $\hat{\theta}_{a}\left(t^{\prime \prime}\right) \in \Omega_{\theta}$. Since $t^{\prime \prime}$ is an arbitrarily chosen time instant of case 2 ), for all $t$ of case 2 ), one has $\hat{\theta}_{a}(t) \in \Omega_{\theta}$.

From the deduction in cases 1) and 2), we have

$$
\hat{\theta}_{a}(t) \in \Omega_{\theta} \quad \forall t \geq 0 .
$$

Noting (38) and (40), from (33), one has $\hat{\theta}(t) \in \Omega_{\theta} \quad \forall t \geq 0$. Thus, the proof of 1) is completed.

Then, we prove 2). Since $\hat{\theta}(t) \in \Omega_{\theta} \quad \forall t \geq 0, \tilde{\theta}=\hat{\theta}-\theta$ is bounded. Therefore, the robust control term satisfying (16) is well defined. Noting (13) and (14), from (16), one has

$$
\begin{aligned}
\dot{V}_{s} & =z_{2} \dot{z}_{2}=z_{2}\left(\theta_{1} u-\theta_{2} x_{2}-\theta_{3} S_{f}-\theta_{4}+\Delta-\dot{x}_{2 \mathrm{eq}}\right) \\
& =z_{2}\left(-\varphi^{\mathrm{T}} \tilde{\theta}+\theta_{1} u_{s 2}-k_{s 1} \frac{\theta_{1}}{\theta_{1 \min }} z_{2}+\Delta\right) \\
& \leq-k_{s 1} z_{2}^{2}+z_{2}\left(-\varphi^{\mathrm{T}} \tilde{\theta}+\theta_{1} u_{s 2}+\Delta\right) \\
& \leq-2 k_{s 1} V_{s}+\varepsilon
\end{aligned}
$$

which leads to (37) in 2).

Remark 2: Similar to the ordinary ARC, the IFARC can guarantee the robust performance in spite of parametric uncertainties and disturbance. Theoretically, the output tracking error can be made arbitrarily small if the design parameter $\varepsilon$ is chosen to be small enough. However, in practice, if $\varepsilon$ is excessively small, the resulting control action will be too large according to (16). As we know, every servomechanism has its input limits. Thus, to keep the control input under limit bounds, the parameter $\varepsilon$ cannot be too small. As a result, the performance bound given by (37) may not meet the performance demand. A less conservative analysis for the performance of IFARC is presented in Theorem 2.

Theorem 2: Assume that $\Delta=0$, i.e., only parametric uncertainties exist. Then, the control system consisting of the IFARC proposed in Section III and the plant (10) satisfies the following.

1) If there is no time instant satisfying (31) and no parameter jump exists after a given time instant $t_{f}$, then the 
following properties hold:

$$
\begin{aligned}
& \tilde{\theta} \in L_{\infty}[0, \infty) \\
& \varepsilon_{e} \in L_{2}[0, \infty) \cap L_{\infty}[0, \infty) \\
& \dot{\hat{\theta}}_{a} \in L_{2}[0, \infty) \cap L_{\infty}[0, \infty) .
\end{aligned}
$$

In addition, if there is a positive number $\xi$ such that

$$
\int_{t-t_{e}}^{t}\left[\hat{\theta}_{\text {ident }}(0)^{\mathrm{T}} \varphi_{2}(p)-f_{\dot{x}_{2}}(p)\right]^{2} d p>\xi \quad \forall t>t_{f}
$$

then the output tracking error $e_{1} \rightarrow 0$ as $t \rightarrow \infty$.

2) If there is any time instant $t_{i}$ satisfying (31) and parameters do not jump in the time interval $\left[t_{i-1}, t_{i}+t_{e}\right]$ but may jump after $t_{i}+t_{e}$, then after $t_{i}+t_{e}$ and before the jump, the parameter estimates are equal to the actual values, i.e., $\hat{\theta}=\theta$; moreover, the output tracking error $e_{1}$ converges to zero exponentially.

Proof: We first prove the result 1) of Theorem 2. From Assumption 1 and the result 1 ) of Theorem 1, it is easy to obtain $\tilde{\theta} \in L_{\infty}[0, \infty)$. From the result 2) of Theorem 1, we know that $x_{2}$ and $u$ are bounded, which leads to the boundedness of $\varphi_{20}$ and $\varphi_{2}$. Combining with (25), we have $\varepsilon_{e} \in L_{\infty}[0, \infty)$. Define a positive definite function $V_{\theta}$ as

$$
V_{\theta}=\frac{1}{2}\left(\hat{\theta}_{a}-\theta\right)^{\mathrm{T}} \Gamma^{-1}\left(\hat{\theta}_{a}-\theta\right) .
$$

If there is no time instant satisfying (30) and no parameter jumps after $t_{f}$, then from (18), we know that, after $t_{f}$, the following holds:

$$
\left\{\begin{array}{l}
\dot{\hat{\theta}}_{a}(t)=\operatorname{Proj}_{\hat{\theta}_{a}}(\Gamma \tau) \\
\tau=\frac{\varphi_{2}}{1+v \varphi_{2}^{T} \varphi_{2}} \varepsilon_{e} .
\end{array}\right.
$$

Noting (19) and (25), from (44), the derivative of $V_{\theta}$ satisfies

$$
\begin{aligned}
\dot{V}_{\theta} & =\tilde{\theta}_{a}^{\mathrm{T}} \Gamma^{-1} \operatorname{Proj}_{\hat{\theta}_{a}}\left(\Gamma \frac{\varphi_{2}}{1+v \varphi_{2}^{\mathrm{T}} \varphi_{2}} \varepsilon_{e}\right) \\
& \leq \frac{\tilde{\theta}_{a}^{\mathrm{T}} \varphi_{2}}{1+v \varphi_{2}^{\mathrm{T}} \varphi_{2}} \varepsilon_{e} \leq-\frac{\varepsilon_{e}^{2}}{1+v \varphi_{2}^{\mathrm{T}} \varphi_{2}}
\end{aligned}
$$

which implies that $\varepsilon_{e} / \sqrt{1+v \varphi_{2}^{\mathrm{T}} \varphi_{2}} \in L_{2}[0, \infty)$. This, along with the boundedness of $\varphi_{2}$, yields

$$
\int_{0}^{t} \varepsilon_{e}^{2} d \tau \leq\left\|1+v \varphi_{2}^{\mathrm{T}} \varphi_{2}\right\|_{\infty} \int_{0}^{t} \frac{\varepsilon_{e}^{2}}{1+v \varphi_{2}^{\mathrm{T}} \varphi_{2}} d \tau<\infty .
$$

Therefore, $\quad \varepsilon_{e} \in L_{2}[0, \infty), \quad$ and then, $\quad \varepsilon_{e} \in L_{2}[0, \infty) \cap$ $L_{\infty}[0, \infty)$. Since $\varphi_{2} /\left(1+v \varphi_{2}^{\mathrm{T}} \varphi_{2}\right)$ and $\varepsilon_{e}$ are bounded, from (44), we get $\dot{\hat{\theta}}_{a} \in L_{\infty}[0, \infty)$. Noting the fact that $\operatorname{Proj}_{\hat{\theta}_{a}}(\Gamma \tau)^{\mathrm{T}} \operatorname{Proj}_{\hat{\theta}_{a}}(\Gamma \tau) \leq(\Gamma \tau)^{\mathrm{T}}(\Gamma \tau)$ [22], from (44) and $\varepsilon_{e} \in L_{2}[0, \infty)$, we have $\dot{\hat{\theta}}_{a} \in L_{2}[0, \infty)$. Hence, we know that $\dot{\hat{\theta}}_{a} \in L_{2}[0, \infty) \cap L_{\infty}[0, \infty)$. Therefore, (42a)-(42c) have been proven.
In the following, we are going to prove $e_{1} \rightarrow 0$ as $t \rightarrow \infty$. First, we will prove $\lim _{t \rightarrow \infty} \varphi_{20}^{\mathrm{T}} \tilde{\theta}_{a}=0$. From (42b) and (42c), one has

$$
\begin{aligned}
& \lim _{t \rightarrow \infty} \varepsilon_{e}=0 \\
& \lim _{t \rightarrow \infty} \dot{\hat{\theta}}_{a}=0 .
\end{aligned}
$$

Applying the swapping lemma [21], [22] and noting (25), the following can be obtained:

$$
\chi=\tilde{\theta}_{a}^{\mathrm{T}} \Omega+\psi+\varsigma
$$

where $\varsigma$ is uniformly bounded, and $\lim _{t \rightarrow \infty} \varsigma(t)=0 ; \chi, \Omega$, and $\psi$ are defined as follows:

$$
\begin{aligned}
& \kappa \dot{\chi}+\chi=\varphi_{20}^{\mathrm{T}} \tilde{\theta}_{a} \\
& \kappa \dot{\Omega}+\Omega=\varphi_{20} \\
& \kappa \dot{\psi}+\psi=-\kappa \Omega^{\mathrm{T}} \dot{\tilde{\theta}}_{a} .
\end{aligned}
$$

According to Theorem 1, it follows that $\varphi_{20}$ is bounded. Therefore, we know that $\Omega$ is also bounded from (48b). In view of (46b) and (48c), one has

$$
\lim _{t \rightarrow \infty} \psi(t)=0 .
$$

From (23), (25), and (48b), we know that $\varepsilon_{e}=-\tilde{\theta}_{a}^{T} \Omega$ for $t>$ $t_{f}$. Hence, from (46a), we have

$$
\lim _{t \rightarrow \infty} \tilde{\theta}_{a}^{\mathrm{T}} \Omega=0 .
$$

Noting that $\lim _{t \rightarrow \infty} \varsigma(t)=0$, from (47), (49), and (50), we obtain

$$
\lim _{t \rightarrow \infty} \chi(t)=0
$$

which yields

$$
\lim _{t \rightarrow \infty} \int_{0}^{t} \dot{\chi}(r) d r=\lim _{t \rightarrow \infty} \chi(t)-\chi(0)=-\chi(0)<\infty .
$$

It is easy to verify that $\chi$ and $\varphi_{20}^{\mathrm{T}} \tilde{\theta}_{a}$ are uniformly continuous. Hence, from (48a), we know that $\dot{\chi}$ is also uniformly continuous. Then, by Barbalat's lemma, $\lim _{t \rightarrow \infty} \dot{\chi}(r)=0$. Since $\varphi_{20}^{\mathrm{T}} \tilde{\theta}_{a}=\kappa \dot{\chi}+\chi$, then

$$
\lim _{t \rightarrow \infty} \varphi_{20}^{\mathrm{T}} \tilde{\theta}_{a}=0 .
$$

If there is no time instant satisfying (31), one has $\hat{\theta}_{\text {ident }}(t)=$ $\hat{\theta}_{\text {ident }}(0)$, where $t>0$. From (32) and (43), we know that

$$
J\left(\hat{\theta}_{\text {ident }}, t\right)>\xi, \quad t \geq 0 .
$$

Noting (46a), from (25) and (32), we have $\lim _{t \rightarrow \infty} J\left(\hat{\theta}_{a}, t\right)=$ 0 , which implies that

$$
J\left(\hat{\theta}_{\text {ident }}, t\right)>J\left(\hat{\theta}_{a}, t\right) \quad \exists t_{\xi}>t_{f} \quad \forall t>t_{\xi} .
$$


According to (33), noting (53) and (54), one has $\hat{\theta}(t)=\hat{\theta}_{a}(t) \quad \forall t>t_{\xi}$. Combining with (52), we have

$$
\lim _{t \rightarrow \infty} \varphi_{20}^{\mathrm{T}} \tilde{\theta}=0 .
$$

Noting that $\theta_{1 \text { min }}>0$, as indicated in Assumption 1, from (15) and (41), we have as follows, when $\Delta=0$ :

$$
\begin{aligned}
\dot{V}_{s} & =z_{2}\left(-\varphi^{\mathrm{T}} \tilde{\theta}+\theta_{1} u_{s}\right)=z_{2}\left(-\varphi^{\mathrm{T}} \tilde{\theta}-\tilde{\theta}_{1} u_{s}+\hat{\theta}_{1} u_{s}\right) \\
& =z_{2}\left(-\varphi_{20}^{\mathrm{T}} \tilde{\theta}+\hat{\theta}_{1} u_{s 2}-k_{s 1} \frac{\hat{\theta}_{1}}{\theta_{1 \min }} z_{2}\right) \\
& \leq-k_{s 1} z_{2}^{2}-z_{2} \varphi_{20}^{\mathrm{T}} \tilde{\theta} \\
& \leq-k_{s 1} V_{s}+\frac{\left(\varphi_{20}^{\mathrm{T}} \tilde{\theta}\right)^{2}}{2 k_{s 1}} .
\end{aligned}
$$

In view of (55) and (56), by applying, Lemma B.8[22], we arrive to the conclusion that $z_{2} \rightarrow 0$ as $t \rightarrow \infty$, which yields $e_{1} \rightarrow 0$ as $t \rightarrow \infty$.

Now we prove the result 2) of Theorem 2. If there is any time instant $t_{i}$ satisfying (31) and a parameter keeps constant in $\left[t_{i-1}, t_{i}+t_{e}\right]$, then after $t_{i}$ and before the parameter jump, $\hat{\theta}_{\text {ident }}(t)=P\left(t_{i}\right)^{-1} Q\left(t_{i}\right)=\theta$, which can be easily verified according to (22) and (27)-(29). Then, after time instant $t_{i}+t_{e}$, $J\left(\hat{\theta}_{\text {ident }}\right) \leq J\left(\hat{\theta}_{a}\right)$. Noting (33), we have $\hat{\theta}=\hat{\theta}_{\text {ident }}=\theta$; thus, $\tilde{\theta}=0$. From (15) and (41), noting that $\tilde{\theta}=0$, if $\Delta=0$, one has

$$
\dot{V}_{s} \leq-k_{s 1} z_{2}^{2}+z_{2}\left(-\varphi^{\mathrm{T}} \tilde{\theta}+\theta_{1} u_{s 2}\right) \leq-2 k_{s 1} V_{s}
$$

which implies that $z_{2}$ converges to zero exponentially, and then, $e_{1}$ converges to zero exponentially. Thus, the proof of Theorem 2 is completed.

Remark 3: It should be noted that the inequality (43) is a very mild condition, since it is almost impossible to find a time interval $\left[t-t_{e}, t\right]$ in which $\hat{\theta}_{\text {ident }}(0)^{\mathrm{T}} \varphi_{2}(t)-f_{\dot{x}_{2}}(t)$ is equal to zero constantly, if $\hat{\theta}_{\text {ident }}(0) \neq \theta(t)\left(t \geq t_{f}\right)$. Because the condition (43) is subtle, from 1) of Theorem 2, we know that, if there is no parameter reset, the output tracking performance is the same as the ordinary indirect ARC (IARC). However, in view of 2) of Theorem 2, we get that, if any parameter reset takes place, the output tracking performance will be improved. In addition, better parameter estimates are available.

Remark 4: If $\Delta \neq 0$, the parameter estimates might be influenced by disturbance. Nevertheless, since the identifier is based on $\int_{t_{i}}^{t_{i+1}} \varphi_{2} \varphi_{2}^{\mathrm{T}} d r \theta=\int_{t_{i}}^{t_{i+1}} \varphi_{2} f_{\dot{x}_{2}} d r$, in which the integral operator is nonsensitive to disturbance, it can provide robust estimation. Furthermore, by the state resets in (27)-(29), the identifier cuts off the forepassed data, so that it can accommodate sudden parameter changes.

Remark 5: From the results of Theorem 2, we can see that $t_{e}$ plays an important role in parameter tuning. Since $t_{e}$ can be regarded as the time delay of parameter jump detection, it should not be too large, so as to guarantee that the IFARC responds to the parameter jump quickly. On the other hand, from (32), we find that $t_{e}$ also relates to the sensitivity of switching logic. The switching may become too sensitive if $t_{e}$ is too small. Therefore, $t_{e}$ should be chosen appropriately.

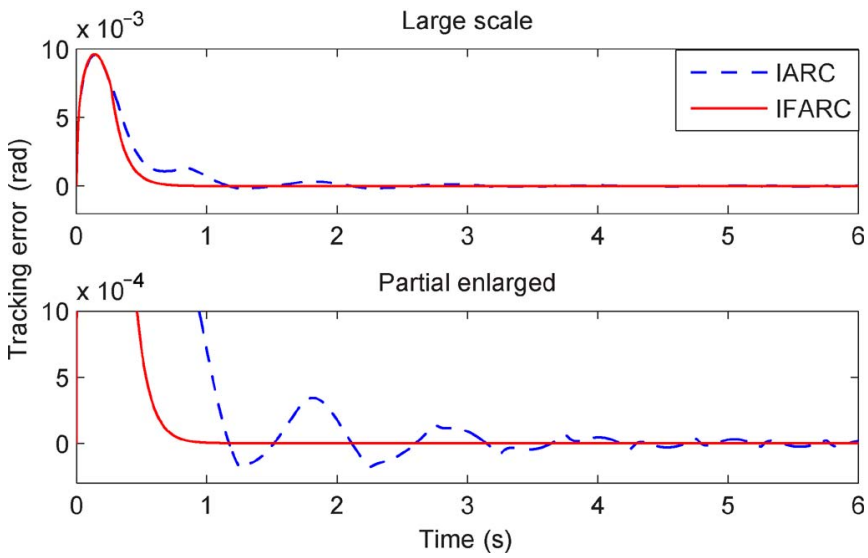

Fig. 3. Output tracking error of IFARC and IARC in Case 1.

\section{Simulations}

In this section, the effectiveness of the proposed method is demonstrated by simulations. Here, two control methods, i.e., the IARC and IFARC, are compared in four cases: 1) constant parameters without disturbance; 2) constant parameters with disturbance; 3) sudden parameter changes without disturbance; and 4) sudden parameter changes with disturbance. The plant $(1-3)$ is applied in the simulations.

\section{A. Case 1: Constant Parameters Without Disturbance}

In this case, no disturbance exists, i.e., $T_{\text {dis }}=0(\mathrm{~N} \cdot \mathrm{m})$. In addition, the plant parameters are constant and given as $J=$ $0.1 \mathrm{~kg} \cdot \mathrm{m}^{2}, B=0.08 \mathrm{~N} \cdot \mathrm{m} /(\mathrm{rad} / \mathrm{s}), T_{c}=0.07 \mathrm{~N} \cdot \mathrm{m}, R_{a}=$ $5 \Omega, K_{T}=5 \mathrm{~N} \cdot \mathrm{m} / \mathrm{A}, K_{E}=0.2 \mathrm{~V} /(\mathrm{rad} / \mathrm{s})$, and $T_{l}=0.1 \mathrm{~N}$. $\mathrm{m}$. Since the ARC design is based on model (10), we should transform the plant (1-3) into the form of model (10), whose parameters are calculated according to (11) and given as

$$
\begin{array}{ll}
\theta_{1}=10\left(\mathrm{rad} / \mathrm{s}^{2}\right) / \mathrm{V} & \theta_{2}=2.8(1 / \mathrm{s}) \\
\theta_{3}=0.7\left(\mathrm{rad} / \mathrm{s}^{2}\right) & \theta_{4}=1\left(\mathrm{rad} / \mathrm{s}^{2}\right) .
\end{array}
$$

It is assumed that the parameter bounds $\theta_{\min }$ and $\theta_{\max }$ are previously known and given as $\theta_{\min }=[5,2,0.5,-10]^{\mathrm{T}}$ and $\theta_{\max }=[15,4,1.5,10]^{\mathrm{T}}$, respectively.

Based on these parameter bounds, IARC and IFARC are designed, respectively. According to the method described in Section III, the controller parameters of IFARC can be designed as $k_{p}=10, k_{s 1}=10, u_{s 2}=-k_{s 2} z_{2} / \theta_{1 \mathrm{~min}}, k_{s 2}=50, \Gamma=$ $\operatorname{diag}([50,20,5,10]), \kappa=0.001, v=1, \varepsilon_{t}=1 \times 10^{-7}, K_{s}=$ $1 \times 10^{6}, t_{e}=0.1 \mathrm{~s}, \gamma=5, \hat{\theta}(0)=[5,3,0.5,10]^{\mathrm{T}}$, and $\hat{\theta}_{a}(0)=$ $\hat{\theta}_{\text {ident }}(0)=\hat{\theta}(0)$. It should be noted that, if the identifier and switching logic component in the IFARC do not work, then the IFARC will become an IARC. That is to say, to design an IARC, we just need to let trig $\equiv 0$ and $\hat{\theta}(t)=\hat{\theta}_{a}(t)$, and then, the parameterized control law will only depend on the parameters given by adaptation.

With the desired trajectory $x_{1 d}=0.1 \sin (2 \pi t)$, the IFARC and IARC are compared by simulations. The curves of output tracking error are shown in Fig. 3. As shown, the tracking error of IFARC is much smaller than that of IARC. That is because 

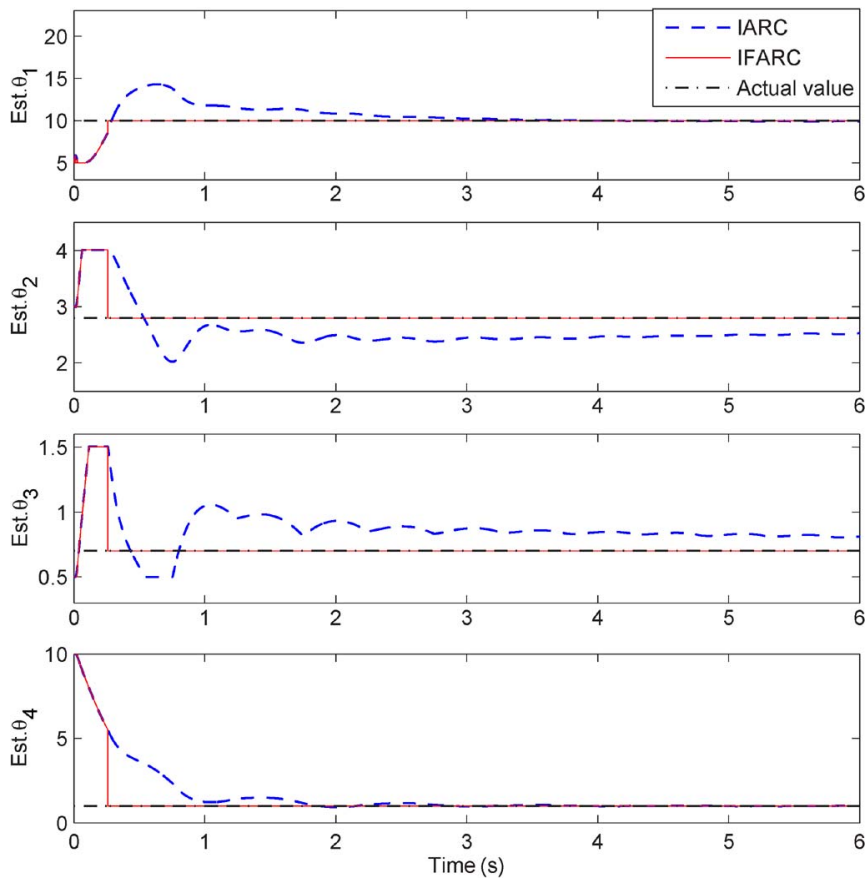

Fig. 4. Parameter estimates of Case 1.

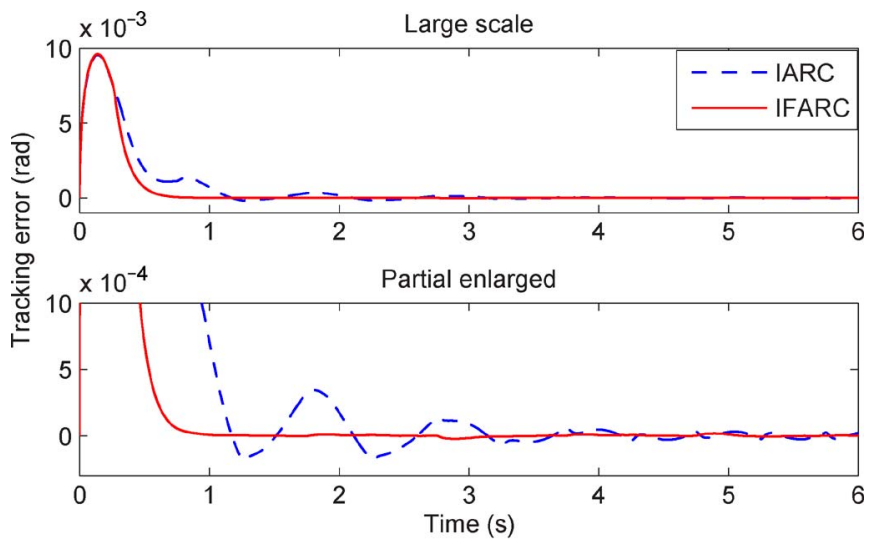

Fig. 5. Output tracking errors of IFARC and IARC in Case 2.

IFARC has better parameter estimates than those of IARC. As shown in Fig. 4, the parameter estimates of IARC converge to the actual values gradually, while IFARC allows the exact reconstruction of the unknown parameters in finite time.

\section{B. Case 2: Constant Parameters With Disturbance}

To test the robustness of IFARC, we apply it to the plant with disturbance. When disturbance exists, the parameter estimation of the adaptation law and identifier would be deteriorated. However, the integral operator embedded in the identifier can attenuate the influence of disturbance; thus, the IFARC can keep in good performance even in the presence of disturbance. In this case, disturbance is added to the plant given in Case 1. The controllers and desired trajectory of this case are the same as those of Case 1 . The disturbance $T_{\text {dis }}$ is a uniformly distributed pseudorandom number with amplitude less than 0.01 . The output tracking errors are shown in Fig. 5. As is demonstrated, the output tracking is just slightly different from that in Case 1.
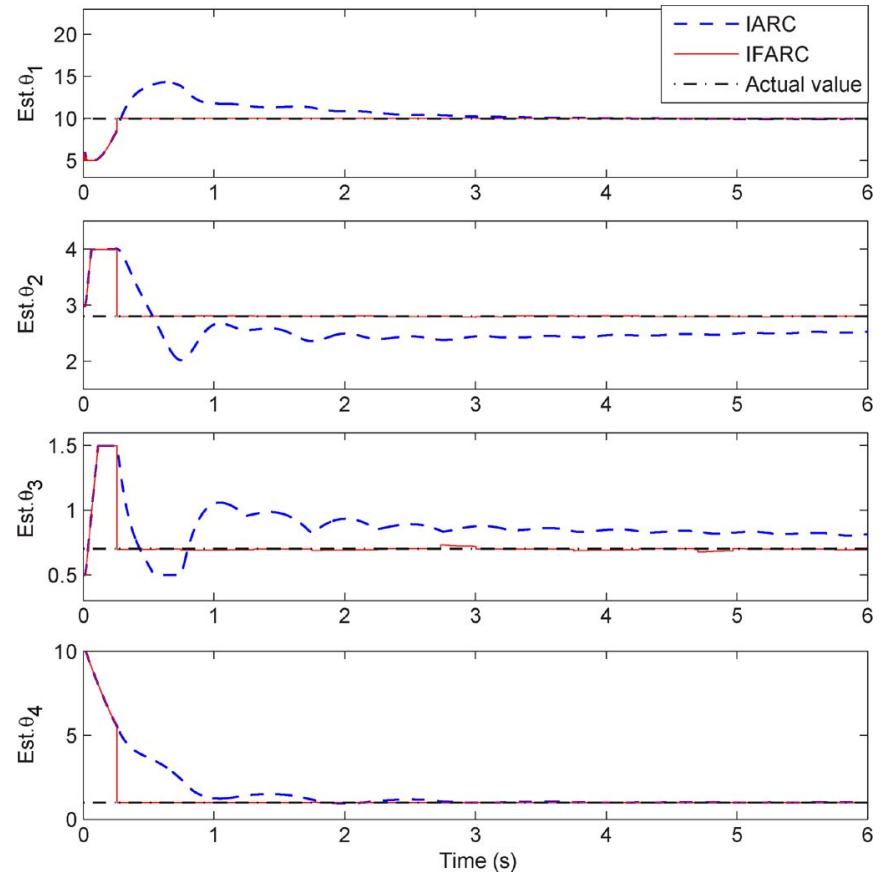

Fig. 6. Parameter estimates of Case 2.

The parameter estimates are shown in Fig. 6. We can see that the parameter estimates of IFARC can get very close to the actual value in finite time, while the estimates of IARC approach the actual value very slowly. Hence, IFARC has better parameter estimation than IARC.

\section{Sudden Parameter Changes Without Disturbance}

The plant with sudden parameter changes and no disturbance is studied in this section. In this case, the plant parameters jump at some time instants, as shown in the following:

$$
\theta= \begin{cases}{[10,2.8,0.7,1]^{\mathrm{T}},} & 0 \leq t<3 \\ {[10,2.8,0.7,4]^{\mathrm{T}},} & 3 \leq t<6 \\ {[8,2.4,0.6,-6.4]^{\mathrm{T}},} & 6 \leq t \leq 9 .\end{cases}
$$

Note that the sudden parameter jumps described in (58) do not exceed the prescribed bounds, i.e., $\theta_{\text {min }} \leq \theta \leq \theta_{\max }$, where $\theta_{\min }$ and $\theta_{\max }$ are given in Section V-A. Therefore, the controllers given in Section $\mathrm{V}-\mathrm{A}$ are also applicable in this case. With the same controllers and input signal as those in Case 1, the simulations are done to demonstrate the effectiveness of the proposed IFARC to the plants with sudden parameter changes. The output tracking error curves are shown in Fig. 7. As we can see, the tracking performance of IFARC is better than that of IARC. In addition, the parameter estimates of IFARC can track the jumping actual values rapidly, as shown in Fig. 8.

Due to the effectiveness of the identifier, the parameter estimates of IFARC can converge to the actual values in finite time. The cooperation of the identifier and adaptation law is shown in Fig. 9. As we can see, at the second identifier reset instant after the parameter jump at $t=3 \mathrm{~s}$ (i.e., the time instant $t_{9}$ ), the parameter estimates jump to the actual values simultaneously. At the first identifier reset instant after the parameter jump (i.e., the time instant $t_{8}$ ), the identifier cannot provide exact estimates 


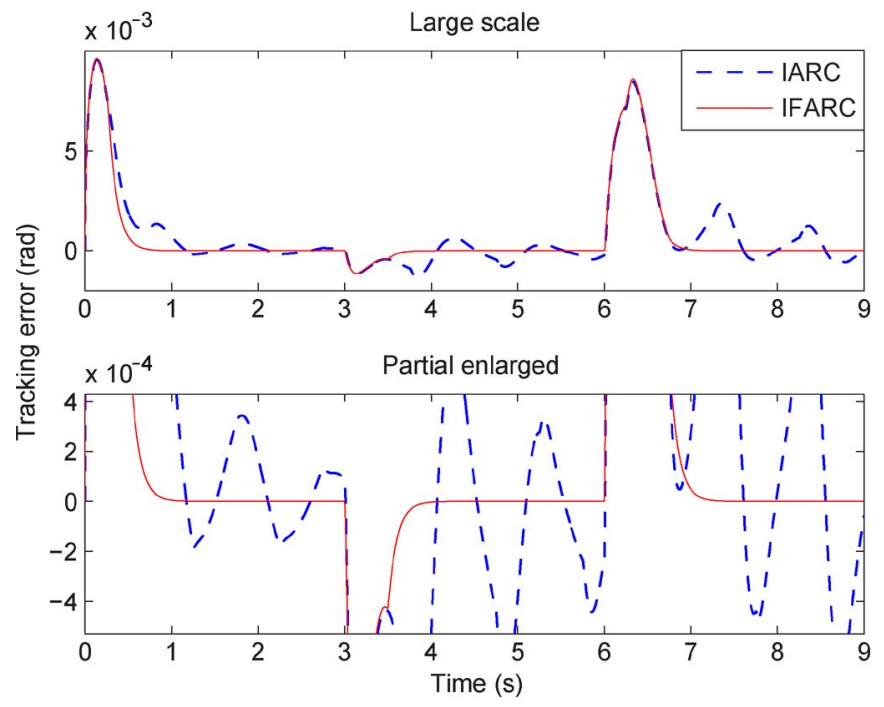

Fig. 7. Output tracking errors of IFARC and IARC in Case 3.
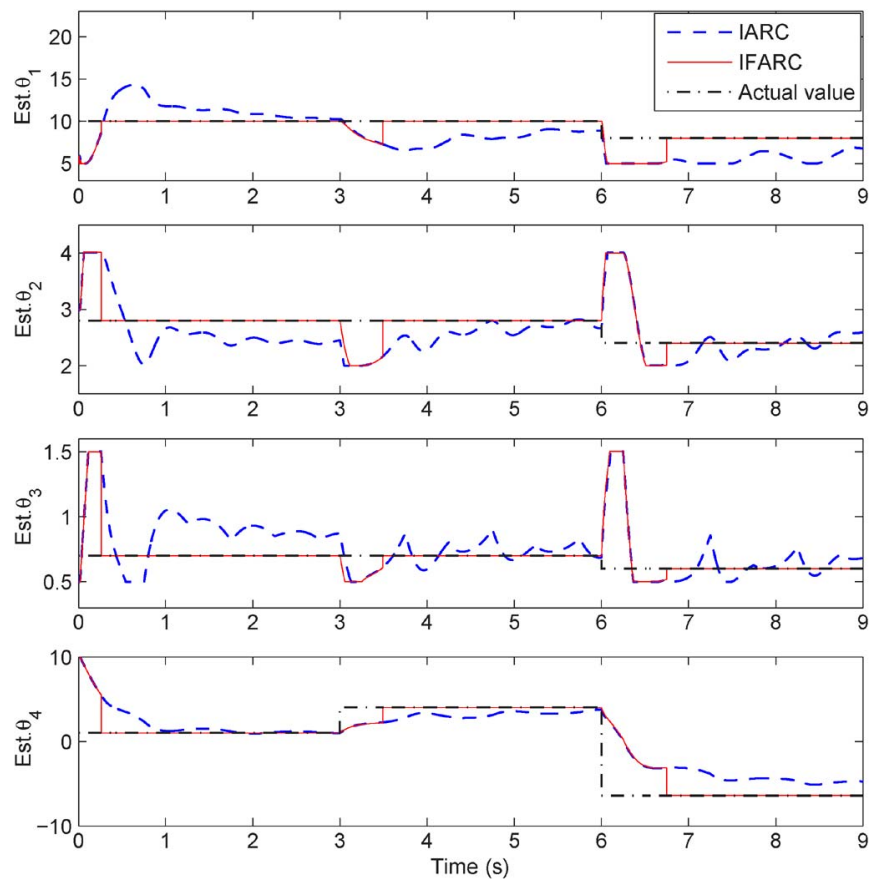

Fig. 8. Parameter estimates of Case 3.

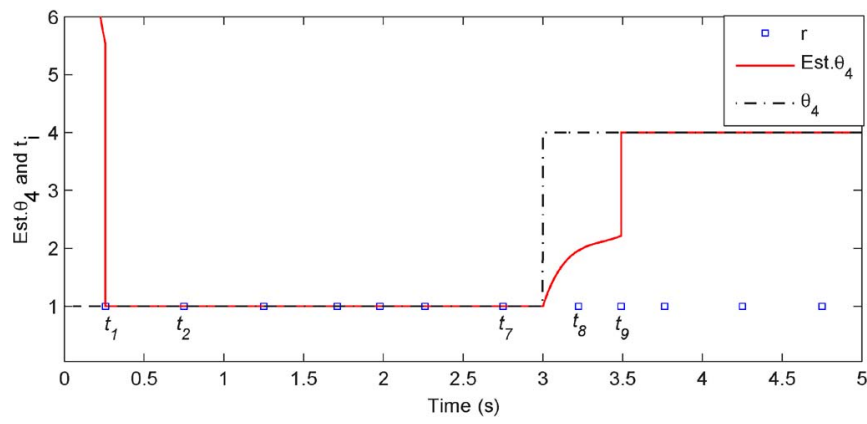

Fig. 9. Illustration of the parameter tuning scheme.

because parameters jump during the interval $\left[t_{7}, t_{8}\right]$, whose data are used to produce identifier estimates at $t_{8}$. Between $t_{7}$ and $t_{8}$, the parameter vector $\hat{\theta}$ is selected between $\hat{\theta}_{a}$ and $\hat{\theta}_{\text {ident }}$ by the

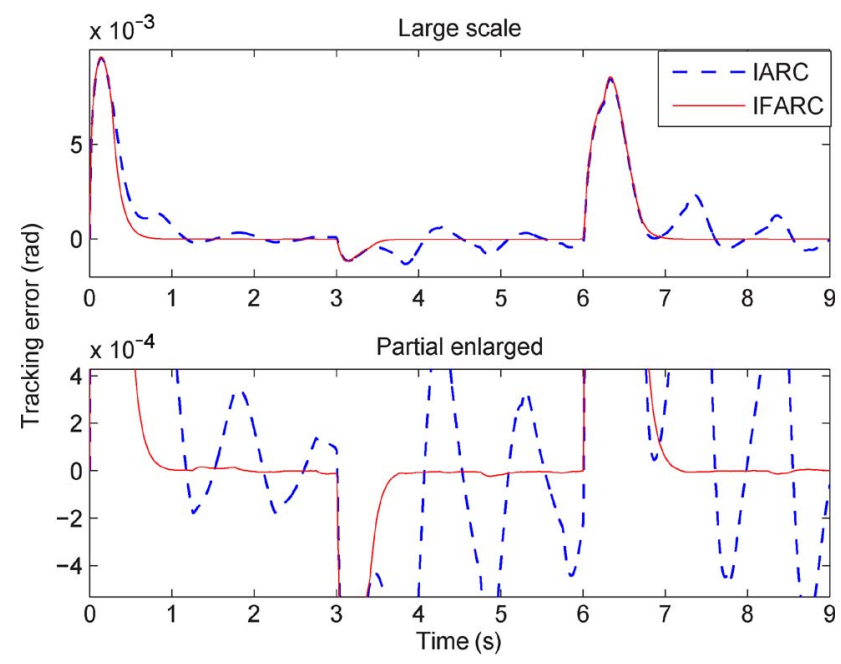

Fig. 10. Output tracking errors of IFARC and IARC in Case 4.
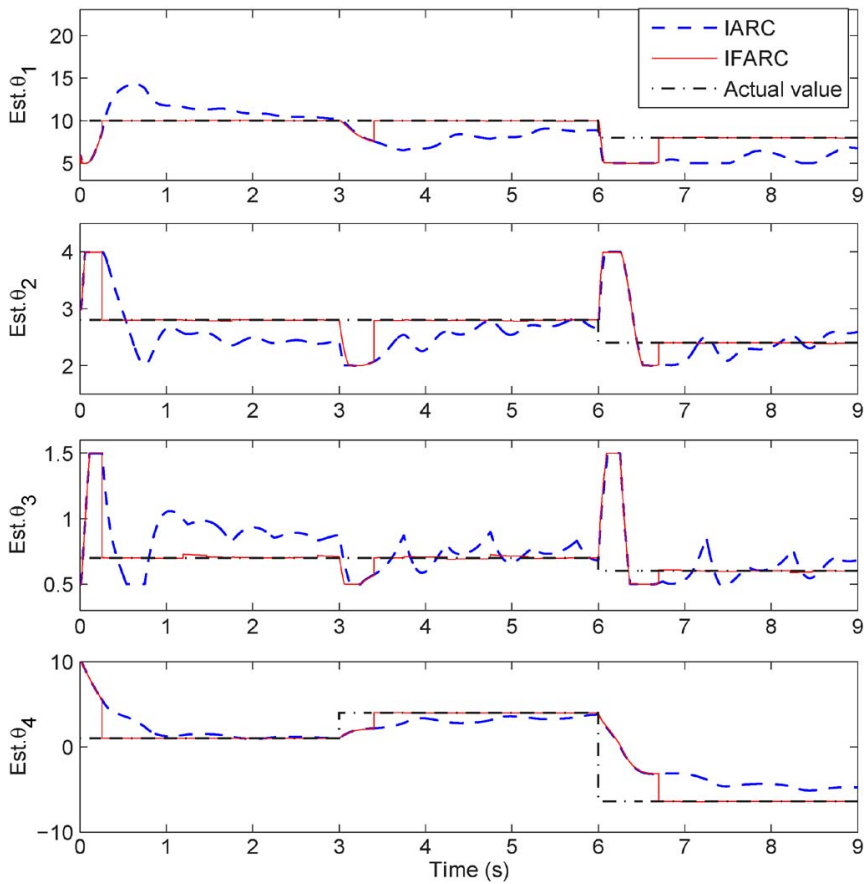

Fig. 11. Parameter estimates of Case 4.

switching logic component. Although the unknown parameters cannot be reconstructed at $t_{8}$, the parameter jump is detected, and the integral operator of the identifier is reset. Since no parameter jump takes place in the time interval $\left[t_{8}, t_{9}\right]$, we have the exact reconstruction of the unknown parameters at $t_{9}$.

\section{Sudden Parameter Changes With Disturbance}

In Case 3, no disturbance exists in the plant. To test the robustness of the proposed IFARC, the same disturbance as in Case 2 is added to the plant in this case. The output tracking error curves of IFARC and IARC are shown in Fig. 10. Their parameter estimates are shown in Fig. 11. We can see that, although the performance of IFARC is slightly affected by the disturbance, it shows much better output tracking performance and parameter estimation than IARC does. Fig. 12 shows the 


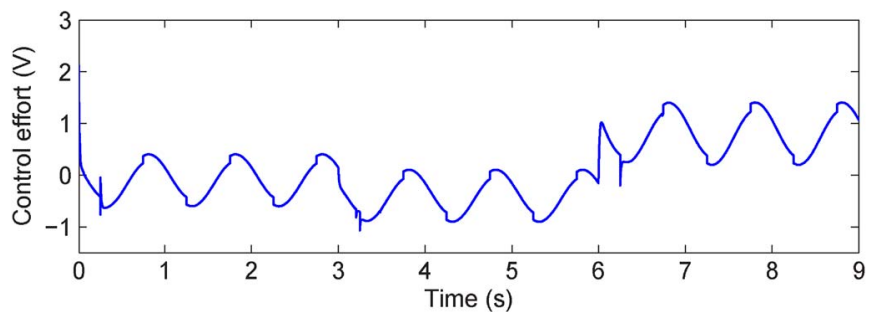

Fig. 12. Control effort of IFARC in Case 4.

TABLE I

PERFORMANCE INDICES OF IARC AND IFARC IN Four CASES

\begin{tabular}{l|ll|ll}
\hline Indices & \multicolumn{2}{|l|}{$L_{2}[e](\mathrm{rad})$} & & $e_{F}(\mathrm{rad})$ \\
\hline controllers & IARC & IFARC & IARC & IFARC \\
\hline Case 1 & 0.00201 & 0.00188 & $3.13 \times 10^{-5}$ & $3.78 \times 10^{-14}$ \\
Case 2 & 0.00202 & 0.00189 & $3.24 \times 10^{-5}$ & $0.96 \times 10^{-6}$ \\
Case 3 & 0.00237 & 0.00226 & 0.0013 & $2.21 \times 10^{-9}$ \\
Case 4 & 0.00238 & 0.00227 & 0.0015 & $5.60 \times 10^{-6}$ \\
\hline
\end{tabular}

control effort trajectory of IFARC in this case. As shown, the IFARC can produce a moderate control action with no chattering even when the plant is subjected to disturbance and parameter sudden changes.

\section{E. Quantitative Analysis}

In order to compare the two controllers in the aforementioned four cases quantitatively, the following performance indices are used [23], [24].

1) $L_{2}[e]=\sqrt{\left(1 / T_{t}\right) \int_{0}^{T_{t}}|e(t)|^{2} d t}$, which is the $L_{2}$ norm of the tracking error, is used as a measure of average tracking performance, where $T_{t}$ represents the total running time.

2) $e_{F}=\max _{t \in \Omega_{t s}}\{|e(t)|\}$, which is the maximum absolute value of the tracking error during the time set $\Omega_{t s}$, within which the system is in steady state. In Cases 1 and 2, the set $\Omega_{t s}$ is defined as $\Omega_{t s}=\left\{t \mid T_{t}-T \leq t \leq T_{t}\right\}$, where $T$ is the period of the input signal $x_{1 d}=0.1 \sin (2 \pi t)$. In Cases 3 and 4, $\Omega_{t s}$ is defined as $\Omega_{t s}=\left\{t \mid T_{i}-T \leq\right.$ $\left.t \leq T_{i}, i=1,2,3\right\}$, where $T_{1}$ and $T_{2}$ represent the time instants of the first and second parameter jumps, respectively; $T_{3}$ represents the total running time in the two cases. $e_{F}$ is used as a measure of final tracking accuracy for periodic trajectories with a period $T$.

The performance indices of the two controllers in four cases are listed in Table I. From the performance indices, we can see that both the average tracking precision and the steady-state tracking precision of IFARC are better than those of IARC.

From the simulation results, we can conclude that the proposed IFARC is effective to enhance the transient performance of the servomechanisms subjected to unknown parameters, disturbances, and parameter sudden changes. This method cannot only provide the desired tracking performance but also has fast and accurate parameter estimation.

\section{CONCLUSION}

In this paper, we have proposed a novel IFARC method. It uses not only an adaptation law but also an embedded identifier to tune the parameters online. A switching logic component has been adopted to select the better parameter vector from the estimate vectors provided by the adaptation law and identifier according to a certain performance index. Then, the control law is constructed with the better parameter vector. The IFARC is applicable to the servomechanisms with parametric uncertainties and disturbance, even with sudden parameter changes. It can guarantee that parameter estimates converge to the actual values in finite time under certain conditions, and then, the desired output tracking performance can be achieved according to the certainty equivalence principle. The stability and control performance of IFARC are analyzed theoretically. Simulations demonstrate that IFARC has better tracking performance and parameter estimation than the IARC method.

\section{REFERENCES}

[1] Z. Yang, K. Kunitoshi, S. Kanae, and K. Wada, "Adaptive robust outputfeedback control of a magnetic levitation system by $K$-filter approach," IEEE Trans. Ind. Electron., vol. 55, no. 1, pp. 390-399, Jan. 2008.

[2] J. Wu and D. Pu, "Adaptive robust motion control of SISO nonlinear systems with implementation on linear motors," Mechatronics, vol. 17, no. 4/5, pp. 263-270, May/Jun. 2007.

[3] J. Zhong and B. Yao, "Adaptive robust precision motion control of a piezoelectric positioning stage," IEEE Trans. Control Syst. Technol., vol. 16, no. 5, pp. 1039-1047, Sep. 2008.

[4] B. Yao, "Integrated direct/indirect adaptive robust control of SISO nonlinear systems in semi-strict feedback form," in Proc. Amer. Control Conf., Denver, CO, 2003, pp. 3020-3025.

[5] C. Y. Lee and J. J. Lee, "Multiple neuro-adaptive control of robot manipulators using visual cues," IEEE Trans. Ind. Electron., vol. 52, no. 1, pp. 320-326, Feb. 2005.

[6] A. Cezayirli and K. Ciliz, "Multiple model based adaptive control of a DC motor under load changes," in Proc. ICM, Istanbul, Turkey, Jun. 2004, pp. $328-333$.

[7] J. A. Farrell and M. M. Polycarpou, Adaptive Approximation Based Control: Unifying Neural, Fuzzy and Traditional Adaptive Approximation Approaches. Hoboken, NJ: Wiley, 2005.

[8] B. Yao and M. Tomizuka, "Adaptive robust control of SISO nonlinear systems in a semi-strict feedback form," Automatica, vol. 33, no. 5, pp. 893900, May 1997.

[9] A. S. Morse, "Towards a unified theory of parameter adaptive control-Part II: Certainty equivalence and implicit tuning," IEEE Trans. Autom. Control, vol. 37, no. 1, pp. 15-29, Jan. 1992.

[10] K. S. Narendra and J. Balakrishnan, "Adaptive control using multiple models," IEEE Trans. Autom. Control, vol. 42, no. 2, pp. 171-187, Feb. 1997.

[11] X. Ye, "Nonlinear adaptive control using multiple identification models," Syst. Control Lett., vol. 57, no. 7, pp. 578-584, Jul. 2008.

[12] A. Cezayirili and M. K. Ciliz, "Indirect adaptive control of non-linear systems using multiple identification models and switching," Int. J. Control, vol. 81, no. 9, pp. 1434-1450, Sep. 2008.

[13] K. K. Tan, S. N. Huang, H. F. Dou, T. H. Lee, S. J. Chin, and S. Y. Lim, "Adaptive robust motion control for precise trajectory tracking applications," ISA Trans., vol. 40, no. 1, pp. 57-71, 2001.

[14] H. Olsson, K. J. Astrom, C. Canudas de Wit, M. Gäfvert, and P. Lischinsky, "Friction models and friction compensation," Eur. J. Control, vol. 4, no. 3, pp. 176-195, 1998.

[15] S. A. Nasar and I. Boldea, Linear Electric Motors: Theory, Design and Practical Applications. Englewood Cliffs, NJ: Prentice-Hall, 1987.

[16] K. S. Narendra and A. M. Annaswamy, Stable Adaptive Systems. Upper Saddle River, NJ: Prentice-Hall, 1989.

[17] V. Adetola, V. Adetola, and M. Guay, "Finite-time parameter estimation in adaptive control of nonlinear systems," IEEE Trans. Autom. Control, vol. 53, no. 3, pp. 807-811, Apr. 2008.

[18] B. Yao, "High performance adaptive robust control of nonlinear systems: A general framework and new schemes," in Proc. 36th IEEE Conf. Decision Control, San Diego, CA, 1997, pp. 2489-2494.

[19] B. Yao and L. Xu, "Adaptive robust motion control of linear motors for precision manufacturing," Mechatronics, vol. 12, no. 4, pp. 595-616, May 2002. 
[20] B. Yao and M. Tomizuka, "Smooth robust adaptive sliding model control of robot manipulators with guaranteed transient performance," Trans. ASME, J. Dyn. Syst. Meas. Control, vol. 118, no. 4, pp. 764-775, 1996.

[21] P. A. Ioannou and J. Sun, Robust Adaptive Control. Upper Saddle River, NJ: Prentice-Hall, 1996.

[22] M. Kristic, I. Kanellakopoulos, and P. V. Kokotovic, Nonlinear and Adaptive Control Design. New York: Wiley, 1995.

[23] L. Xu and B. Yao, "Adaptive robust precision motion control of linear motors with negligible electrical dynamics: Theory and experiments," IEEE Trans. Mechatronics, vol. 6, no. 4, pp. 444-452, Dec. 2001.

[24] L. Lu, Z. Chen, B. Yao, and Q. Wang, "Desired compensation adaptive robust control of a linear-motor-driven precision industrial gantry with improved cogging force compensation," IEEE Trans. Mechatronics, vol. 13, no. 6, pp. 617-624, Dec. 2008.

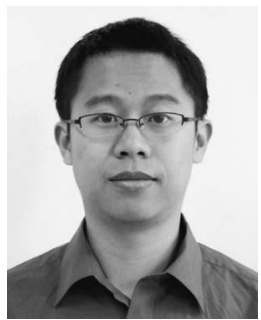

Guozhu Zhang was born in Guangdong, China, in 1984. He received the B.S. degree in automation from Beijing Institute of Technology, Beijing, China, in 2004, where he is currently working toward the Ph.D. degree in control theory and control engineering.

His main research interests include adaptive control, servomechanism, robust control, and nonlinear system identification.

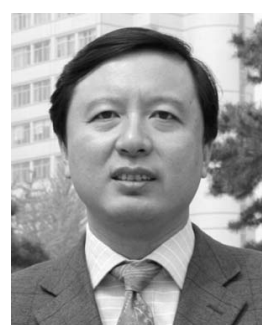

Jie Chen (M'09) received the B.S., M.S., and Ph.D. degrees in control theory and control engineering from Beijing Institute of Technology, Beijing, China, in 1986, 1993, and 2000, respectively.

From 1989 to 1990, he was a Visiting Scholar with The California State University, Los Angeles. From 1996 to 1997, he was a Research Fellow with the School of Electronic and Electrical Engineering, University of Birmingham, Birmingham, U.K. He is currently a Professor with the School of Automation, Beijing Institute of Technology. His main research interests include multiindex optimization, multiobjective decision and control, intelligent control, and constrained nonlinear control.

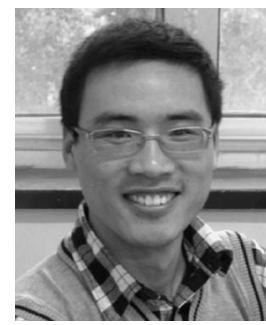

Zhiping Li was born in Hunan, China, in 1984. $\mathrm{He}$ received the B.S. degree in automation from Beijing Institute of Technology, Beijing, China, in 2005 , where he is currently working toward the Ph.D degree in control theory and control engineering.

His main research interests include adaptive control, neural network control, and motion control. 